Article

\title{
Green Tea Extract Induces the Resistance of Caenorhabditis elegans against Oxidative Stress
}

\section{Sami Abbas ${ }^{1}$ and Michael Wink ${ }^{2, *}$}

1 Department of Biotechnology, Faculty of Applied Sciences, University of Kalamoon, Damascus countryside, Deir Attiah, Syria; E-Mail: sami.abbas@uok.edu.sy

Institute of Pharmacy and Molecular Biotechnology, Heidelberg University, Im Neuenheimer Feld 364, Heidelberg 96120, Germany

* Author to whom correspondence should be addressed; E-Mail: wink@uni-hd.de; Tel.: +49-6221-544-881; Fax: +49-6221-544-884.

Received: 20 January 2014; in revised form: 13 February 2014 / Accepted: 19 February 2014 / Published: 4 March 2014

\begin{abstract}
Epidemiological studies on the effects of green tea consumption (Camellia sinensis) have demonstrated a reduction for the risk of age-related diseases. The investigation of the in vivo and in vitro antioxidant properties of an aqueous extract of green tea (GTE) was the aim of the current study. 2,2-Diphenyl-1-picrylhydrazyl (DPPH*) and superoxide anion radical $\left(\mathrm{O}_{2}{ }^{-}\right)$assays were used to estimate the GTE antioxidant activity. To investigate the protective effects of GTE against oxidative stress, wild-type N2 and transgenic strains (TJ374, hsp-16.2/GFP) of the model organism, Caenorhabditis elegans ( $C$. elegans), were chosen. In the current study, the following catechins were identified by LC/ESI-MS: catechin, epicatechin, epicatechin gallate, gallocatechin, epigallocatechin and epigallocatechin gallate. GTE exhibited a free radical scavenging activity of $\mathrm{DPPH}^{\circ}$ and $\mathrm{O}_{2}{ }^{--}$with $\mathrm{IC}_{50} 8.37$ and $91.34 \mu \mathrm{g} / \mathrm{mL}$, respectively. In the C. elegans strain (TJ374, $h s p-16.2 / G F P$ ), the expression of $h s p-16.2 / G F P$ was induced by a nonlethal dose of juglone, and the fluorescence density of $h s p-16.2 / G F P$ was measured. The $h s p-16.2 / G F P$ was reduced by $68.43 \%$ in the worms pretreated with $100 \mu \mathrm{g} / \mathrm{mL}$ GTE. N2 worms pretreated with $100 \mu \mathrm{g} / \mathrm{mL}$ GTE exhibited an increased survival rate of $48.31 \%$ after a lethal dose application of juglone. The results suggest that some green tea constituents are absorbed by the worms and play a substantial role to enhance oxidative stress resistance in C. elegans.
\end{abstract}


Keywords: LC/ESI-MS; catechins; antioxidants; DPPH; superoxide anion radical; hsp-16.2/GFP

\section{Introduction}

Reactive oxygen species (ROS) are important molecules in biological systems, which are produced as by-products of normal metabolism. ROS include radical molecules, such as superoxide anion, and non-radical molecules, such as $\mathrm{H}_{2} \mathrm{O}_{2}$. Peroxisomes contribute to $\mathrm{H}_{2} \mathrm{O}_{2}$ production, which use it to oxidize a variety of molecules. On the other hand, peroxisomes contain catalase, which degrades $\mathrm{H}_{2} \mathrm{O}_{2}$ to $\mathrm{H}_{2} \mathrm{O}$ and $\mathrm{O}_{2}$. However, in the case of the overproduction of $\mathrm{H}_{2} \mathrm{O}_{2}$ or when peroxisomes are damaged, cells can become exposed to oxidative stress. Overproduction of free radicals and ROS can damage important cellular macromolecules (DNA, proteins and biomembranes), which would contribute to ROS- and age-related diseases, including cancer and neurodegenerative and cardiovascular diseases [1-5].

Camellia sinensis L. Kuntze (Theaceae) has been used for medicinal purposes for several centuries. Today, it is a widely consumed beverage throughout the world and cultivated commercially in Asia, Africa and South America [6]. Green tea and its constituents exhibit a broad array of biological activities. The most important green tea constituents are the catechins, a group of bioactive polyphenols. They include epigallocatechin gallate (EGCG), epicatechin gallate (ECG), gallocatechin (GC), epigallocatechin (EGC), epicatechin (EC) and catechin (C) [7]. EGCG is one of the major components in green tea [8], and its pharmacology has been intensively studied [9-15].

Green tea polyphenols showed positive effects for cardiovascular diseases and as cancer chemopreventive agents [16-22]. Several studies have demonstrated that green tea acts as an anti-inflammatory agent [23]. Green tea consumption can improve metabolic biomarkers [24] and lower body weight [25]. There is good evidence that the beneficial effects of green tea result from the antioxidant activities of its polyphenols, which are able to scavenge free radicals and reactive oxygen species [26-28]. Furthermore, polyphenols can dissociate into phenolates with $\mathrm{O}^{-}$ions under physiological conditions. The hydroxyl groups and $\mathrm{O}^{-}$ions can form hydrogen and ionic bonds with multiple proteins and nucleic acids in the body. This interaction influences protein flexibility, conformation and, as a consequence, their bioactivity. Therefore, they can modulate various proteins (enzymes, receptors and transcription factors) that play a role in health disorders and diseases [29-31].

The versatile model nematode, C. elegans Maupas (Rhabditidae), is useful for understanding the effects of pro-oxidants and antioxidants and their mode of action, because the nematodes have a rapid life cycle, short lifespan, well-established genetic pathways and are easy to cultivate [28,32]. Because C. elegans shares a substantial number of genes and pathways with humans, these nematodes have become an interesting pharmacological model organism [33-35].

EGCG is one of the most active and bioavailable antioxidant in green tea [36]. Our previous results showed that EGCG induces longevity and protects the model organism C. elegans against oxidative stress induced by the pro-oxidant naphthoquinone, juglone [27,28]. EGCG probably acts through the daf2/insulin-signaling pathway, because EGCG induces a translocation of the transcription factor 
DAF-16 from cytoplasm into nucleus in the TJ356 (DAF-16/GFP) strain [37]. Catechin, another constituent from green tea, can extend the lifespan in $C$. elegans and increase its stress resistance by modulating the energy-intensive stress response and repair system [38], which is in agreement with the Disposable Soma Theory [39]. Furthermore, the phenolics of resveratrol, aspalathin from Aspalathus linearis and anthocyanin-rich purple wheat increased longevity and resistance against oxidative stress in C. elegans [40-42]. The effects of aspalathin and anthocyanin-rich purple wheat might be mediated via a regulation of the DAF-16/FOXO insulin-like signaling pathway [40,42]. Blueberry polyphenols prolong the lifespan [43] through the CaMKII pathway, which mediates osmotic stress resistance [44]. Ginkgo biloba EGb761 protects the worms against oxidative damage, which accumulates upon aging [45]. Valproic acid prolongs the lifespan by inhibiting the activity of the insulin/IGF-1 signaling pathway, in addition to other targets [46].

The present study aimed to investigate the ability of an aqueous extract of green tea (GTE) to scavenge the free radicals in vitro and to elucidate the effect of GTE treatment on oxidative stress resistance in $C$. elegans. The transgenic strain, TJ374 ( $h s p-16.2 / G F P)$, of the model organism, C. elegans, was used to investigate the ability of GTE to be absorbed by $C$. elegans and to protect the worms against oxidative stress. This strain is a useful tool to monitor the oxidative stress effects in living organism, since $h s p-16.2 / G F P$ is used as a marker for the oxidative stress.

\section{Experimental Section}

\subsection{Chemicals and Plant Material}

Japanese green tea was purchased from Paul Schrader GmbH \& Co. KG, Bremen, Germany. Sixty grams of dried leaves of green tea were immersed into $2 \mathrm{~L}$ of water overnight at $40{ }^{\circ} \mathrm{C}$. The water extract was collected, filtered and finally lyophilized. For our experiments, the powder was dissolved in sterilized and bidistilled water. For in vitro tests, a range of GTE concentrations was used that allows one to draw linear curves and to calculate the $\mathrm{IC}_{50}$. For in vivo experiments, the GTE concentration is chosen depending on the curves resulting from in vitro study, since the concentration of $100 \mu \mathrm{g} / \mathrm{mL}$ of GTE was almost in the plateau part of the curves (Figure 3). (-)-Epigallocatechin gallate (EGCG) 95\%, 2,2-diphenyl-1-picrylhydrazyl (DPPH'), nicotinamide adenine dinucleotide (NADH), phenazine methosulfate (PMS) and 5-hydroxy-1, 4-naphthoquinone (juglone) were obtained from Sigma-Aldrich GmbH (Steinheim, Germany). Nitroblue tetrazolium (NBT) and sodium azide came from AppliChem GmbH (Darmstadt, Germany).

\subsection{C. elegans Strains and Culture Conditions}

The TJ375 (hsp-16.2/GFP) and N2 strains were obtained from the Caenorhabditis Genetic Center (CGC), which was founded by NIH National Center for Research Resources. The strains were maintained at $20{ }^{\circ} \mathrm{C}$ on solid nematode growth medium (NGM) [32]. All worms used in this study were age-synchronized, and the experimental animals were grown in liquid S-medium and raised from eggs obtained by sodium hypochlorite treatment of hermaphrodites [47]. 


\subsection{Analysis of Catechins in GTE Using the LC/ESI-MS}

The HPLC system consists of a degasser, Merck-Hitachi L-6200A low pressure binary pumps and Lichro-CART RP-C18 e column $(250 \times 4.6 \mathrm{~mm}$ i.d.; $5 \mu \mathrm{m})$ with a compatible guard column. The mobile phase was operated under gradient condition with $0.5 \%$ aqueous acetic acid (A) and acetonitrile (B), at a flow rate $1 \mathrm{~mL} / \mathrm{min}$ at room temperature as follows: 0-60 $\min 0 \%-40 \% \mathrm{~B}, 60-70 \mathrm{~min}$ $40 \%-100 \%$ B. The MS machine used was a VG Quattro II model with an electrospray source and a quadrupole analyzer. Acquisition was done in negative mode using single-quad with the following parameters: drying and nebulizing gas $\left(\mathrm{N}_{2}\right)$ with a flow rate of $3.5 \mathrm{~L} / \mathrm{h}$ and $350 \mathrm{~L} / \mathrm{h}$, respectively; source temperature, $120^{\circ} \mathrm{C}$; scan range, 200-1000 m/z; cone voltage, $30 \mathrm{~V}$; focusing voltage, $0.5 \mathrm{~V}$; and capillary voltage, $3.5 \mathrm{kV}$.

\subsection{DPPH Free Radical Scavenging Activity}

The free radical scavenging activity of GTE was measured using 2,2-diphenyl-1-picrylhydrazyl $\left(\mathrm{DPPH}^{\circ}\right)$, following the standard methods [48-50] with some modifications. The antioxidants reduce the stable free radical DPPH (deep violet) to the diphenyl-picrylhydrazine DPPH-H (pale yellow). The $\mathrm{DPPH}^{\circ}$ molecule is a stable free radical characterized by an absorption band at about $517 \mathrm{~nm}$. As the odd electron of the radical becomes paired in the presence of a hydrogen donor (antioxidant), the absorption strength is decreased [49,51]. Zero-point-two millimolar of $\mathrm{DPPH}^{*}$ in methanol was prepared, and $500 \mu \mathrm{L}$ of this solution was added to $500 \mu \mathrm{L}$ of GTE at different concentrations that allow one to draw linear curves and to calculate the $\mathrm{IC}_{50}$. Thirty minutes later, the absorbance was measured at $517 \mathrm{~nm}$. The scavenging activity of the $\mathrm{DPPH}^{\circ}$ free radicals (in \%) was calculated using the following equation:

$$
\mathrm{DPPH}^{\bullet} \text { scavenging effect }(\%)=\left[\left(A_{0}-A_{1}\right) / A_{0}\right] \times 100
$$

$A_{0}$ is the absorbance of the control reaction ( $\mathrm{DPPH}^{\circ}$ reagent without sample), and $A_{1}$ is the absorbance in the presence of GTE. EGCG was employed as a positive control. Data were obtained from three independent experiments. The mean and standard error were calculated for each concentration.

\subsection{Superoxide Anion Radical Scavenging Activity}

The superoxide anion scavenging activity of GTE was determined by the method of Robak and Gryglewski [52]. Superoxide anion radicals are generated in the PMS-NADH system by oxidation of $\mathrm{NADH}$ and assayed by the reduction of NBT. The superoxide radicals were generated in $900 \mu \mathrm{L}$ of $0.1 \mathrm{M}$ phosphate buffer, $\mathrm{pH} 7.4$ (reference), or $800 \mu \mathrm{L}$ (test), $100 \mu \mathrm{L}$ water (control) or GTE at different concentrations that allow one to draw linear curves and to calculate the $\mathrm{IC}_{50}, 100 \mu \mathrm{L}$ NADH $(156 \mu \mathrm{M})$ and $100 \mu \mathrm{L}$ NBT $(630 \mu \mathrm{M})$. The reaction starts by adding $100 \mu \mathrm{L}$ PMS $(30 \mu \mathrm{M})$ to the mixture. After $5 \mathrm{~min}$ of incubation at $25{ }^{\circ} \mathrm{C}$, the absorption was measured at $560 \mathrm{~nm}$. The percentage of inhibition of superoxide anion generation was calculated using the following equation:

$$
\mathrm{O}_{2}^{-\bullet} \text { scavenging effect }(\%)=\left[\left(A_{0}-A_{1}\right) / A_{0}\right] \times 100
$$


$A_{0}$ is the absorbance of the control reaction, and $A_{1}$ is the absorbance in the presence of GTE. EGCG was employed as a positive control. Data were obtained from three independent experiments. The mean and standard error were calculated for each concentration.

\subsection{Quantitation of hsp-16.2/GFP Expression in C. elegans}

TJ375 (hsp-16.2/GFP) worms were cultivated in liquid S-medium at $20^{\circ} \mathrm{C}$, and E. coli (OP50) was added to the media $\left(1 \times 10^{9} \mathrm{cell} / \mathrm{mL}\right)$ as a food source. $h s p-16.2$ is expressed by either heat shock or by pro-oxidative stress, like exposure to the naphthoquinone, juglone [53]. The expression of $h s p-16.2$ was measured directly by observing the fluorescence of the green fluorescent protein (GFP) as the reporter protein. The worms were treated with $100 \mu \mathrm{g} / \mathrm{mL}$ of GTE on the day after hatching for $48 \mathrm{~h}$. Worms were transferred to new medium, and oxidative stress was then exerted by adding $20 \mu \mathrm{M}$ juglone to the medium for $24 \mathrm{~h}$. After induction, worms from each set of experiments were mounted onto a glass slide in M9 medium containing $10 \mathrm{mM}$ sodium azide to reduce their mobility [47]. Images of fluorescence were taken at constant exposure times using the $20 \times$ objective (Nikon-eclipse 90i, Nikon Imaging Center, Heidelberg University) with a digital camera (Nikon digital sight DS-Qi1Mc). The images included the anterior part of worms from the back of the pharynx. For quantifying the GFP fluorescence, images were analyzed using ImageJ software. The image of the anterior part of the worms was outlined, black to white inverted and the mean pixel density measured.

\subsection{Survival Assay}

Through induced oxidative stress, a higher concentration of juglone $(80 \mu \mathrm{M})$ is lethal. N2 worms were maintained at $20{ }^{\circ} \mathrm{C}$ and treated with $100 \mu \mathrm{g} / \mathrm{mL}$ GTE on the day after hatching for $48 \mathrm{~h}$, and then, worms were transferred to new medium. Eighty micromolar juglone was added to the medium, and the survivors were counted after $24 \mathrm{~h}$ of juglone treatment.

\subsection{Statistical Analyses}

Statistical comparison between controls and treatments were done with two-tailed unpaired student's $t$-test, assuming equal variance. All figures indicate means and the standard error of the mean. Data were obtained from three independent experiments. Error bars represent the standard error (SE). $p<0.05$ was considered for significant differences.

\section{Results and Discussion}

\subsection{Catechins of GTE}

Several studies have applied LC/ESI-MS to identify the main secondary metabolites of green tea, especially of catechins (flavan-3-ols), using retention time and mass spectral information [7,54-56]. A reconstructed ion chromatogram (RIC) of the aqueous extract is shown in Figure 1. MS analysis allowed the identification of catechins by the presence of informative $[\mathrm{M}-\mathrm{H}]^{-}$ions, along with a unique retention time for each component. The mass spectrum of EGCG shows a fragment ion at $\mathrm{m} / \mathrm{z} 287$ resulting from the loss of water from the EGC moiety, and $m / z 305$ represents the EGC moiety 
(Figure 2b, Table 1). The mass spectrum of ECG shows a fragment ion at $\mathrm{m} / \mathrm{z} 271$ resulting from the loss of water from the epicatechin moiety (Figure 2b, Table 1). Mass spectral data are partly in agreement with the previous results of Poon [55], because he obtained the full scan negative ionization mass spectra, while in our case, the scan range was $200-1000 \mathrm{~m} / \mathrm{z}$. Figure 2a,b illustrates the mass spectra and structures of catechin, epicatechin, gallocatechin, epigallocatechin, epicatechin gallate and epigallocatechin gallate with $[\mathrm{M}-\mathrm{H}]^{-}$at $\mathrm{m} / \mathrm{z} 289,289,305,305,441$ and 457, respectively; these findings are in agreement with published results [7,54]. The molecular ions and some informative fragments of GTE catechins are summarized in Table 1.

Figure 1. LC/ESI-MS analysis of aqueous extract of green tea (GTE). Reconstructed ion chromatogram (RIC) obtained in both positive and negative scan modes. GC, gallocatechin; EGC, epigallocatechin; C, catechin; EC, epicatechin; EGCG, epigallocatechin gallate; ECG, epicatechin gallate.

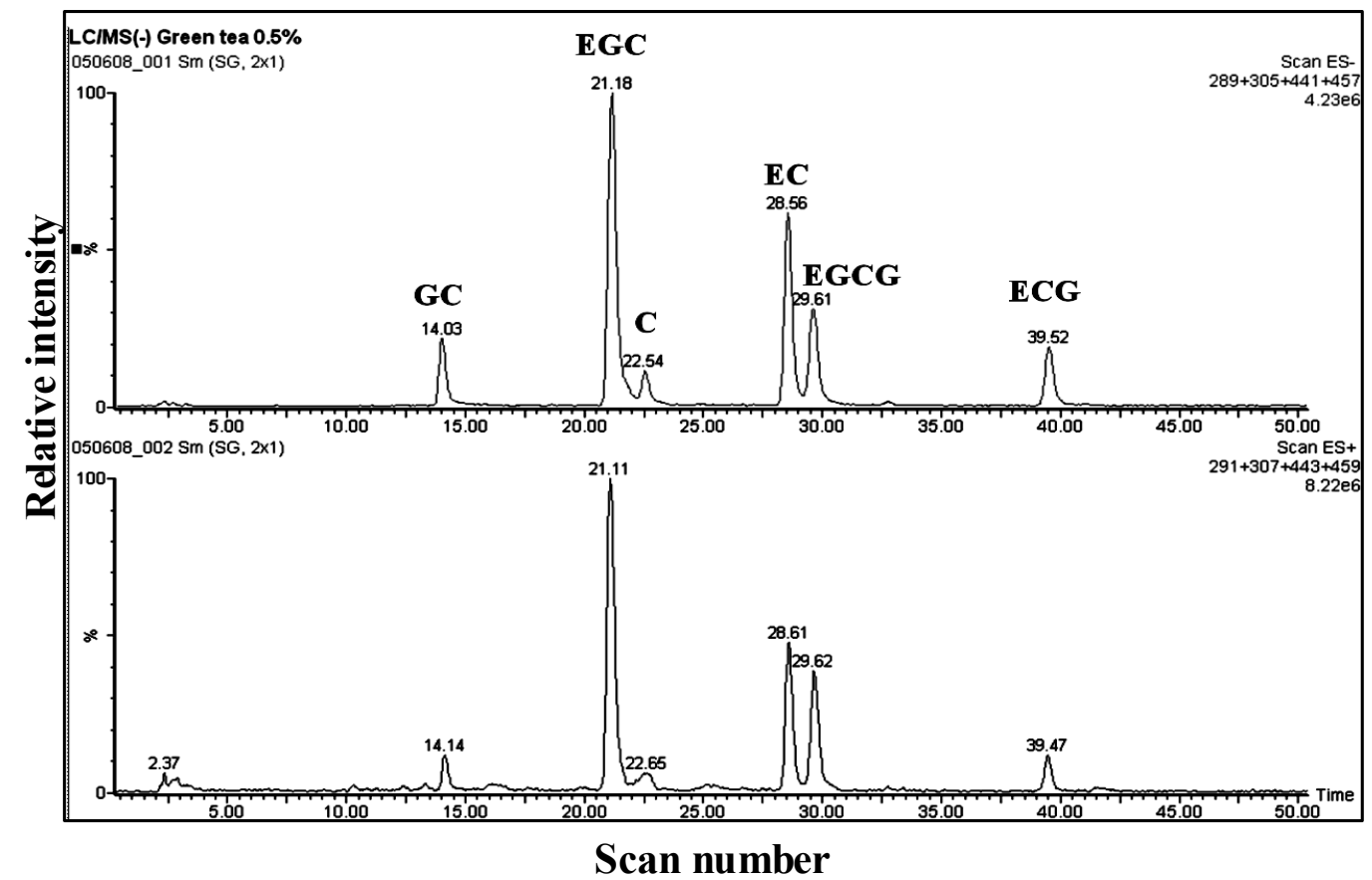

Table 1. Molecular ions and some significant fragments of main catechins presented in GTE.

\begin{tabular}{cccc}
\hline Compound & MW & {$[\mathbf{M}-\mathbf{H}]^{-}$} & Significant fragments \\
\hline Catechin (C) & 290 & 289 & \\
Epicatechin (EC) & 290 & 289 & \\
Gallocatechin (GC) & 306 & 305 & \\
Epigallocatechin (EGC) & 306 & 305 & \\
Epicatechin gallate (ECG) & 442 & 441 & $\mathrm{~m} / \mathrm{z} 271\left(\mathrm{EC}\right.$ without $\left.\mathrm{H}_{2} \mathrm{O}\right) \mathrm{m} / \mathrm{z} 289(\mathrm{EC})$ \\
Epigallocatechin gallate (EGCG) & 458 & 457 & $\mathrm{~m} / \mathrm{z} 287\left(\mathrm{EGC}\right.$ without $\left.\mathrm{H}_{2} \mathrm{O}\right) \mathrm{m} / \mathrm{z} 305$ (EGC) \\
\hline
\end{tabular}


Figure 2. Mass spectra of catechins. (a) Catechin with $[\mathrm{M}-\mathrm{H}]^{-}$of $\mathrm{m} / z 289$, epigallocatechin with $[\mathrm{M}-\mathrm{H}]^{-}$of $\mathrm{m} / \mathrm{z} 305$ and gallocatechin with $[\mathrm{M}-\mathrm{H}]^{-}$of $\mathrm{m} / \mathrm{z} 305$; (b) epicatechin gallate with $[\mathrm{M}-\mathrm{H}]^{-}$of $m / z 441$, epigallocatechin gallate with $[\mathrm{M}-\mathrm{H}]^{-}$of $m / z 457$ and epicatechin with $[\mathrm{M}-\mathrm{H}]^{-}$of $m / z 289$.
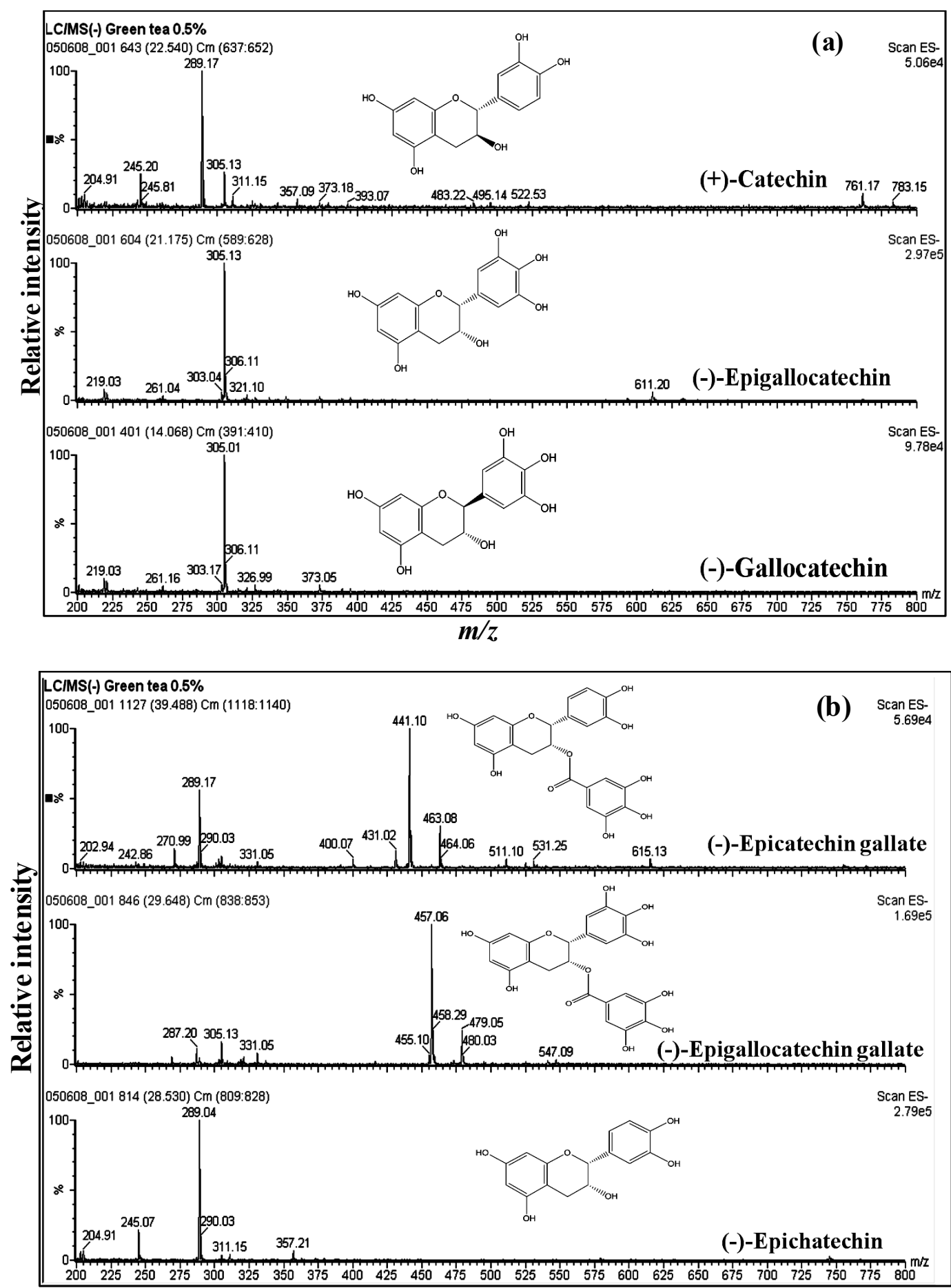

$m / z$

\subsection{GTE Scavenges $\mathrm{DPPH}$ and $\mathrm{O}_{2}{ }^{--}$Free Radicals in Vitro}

The excessive oxidation reactions may occur from exposure to stress, smoking and chemical reagents, resulting in the production of free radicals that can contribute to the onset of various disease states, 
such as cardiovascular diseases, cancer and neurological disorders, and also accelerate aging [1,2,57-59]. GTE showed a strong free radical scavenging activity with an $\mathrm{IC}_{50}$ of $8.37 \pm 0.14 \mu \mathrm{g} / \mathrm{mL}$ (Figure 3). The superoxide anion was generated in a non-enzymatic system using PMS, NADH and NBT. The decrease of absorbance at $560 \mathrm{~nm}$ with GTE indicates a consumption of superoxide anion in the reaction mixture with an $\mathrm{IC}_{50}$ of $91.34 \pm 1.98 \mu \mathrm{g} / \mathrm{mL}$ (Figure 3). These results are in agreement with our earlier studies on EGCG [27] and those of other reports [8,31,60-63].

Figure 3. Two assays were used to measure the in vitro antiradical activities of GTE. Free radical scavenging activity was determined by the 2,2-diphenyl-1-picrylhydrazyl (DPPH') assay and superoxide anion radical assay. The superoxide anion radicals were generated in the phenazine methosulfate (PMS)-nicotinamide adenine dinucleotide (NADH) system by oxidation of NADH and assayed by the reduction of nitroblue tetrazolium (NBT). The results of these tests are expressed as the percent inhibition of the control (reagent without sample) that is calculated by Equations (1) and (2). For the $\mathrm{DPPH}^{\circ}$ assay, the range of GTE concentrations used was $2-24 \mu \mathrm{g} / \mathrm{mL}$. For the superoxide assay, the range of GTE concentrations used was $10-120 \mu \mathrm{g} / \mathrm{mL}$. Data were obtained from three independent experiments. The mean and standard error were calculated for each concentration. $\mathrm{IC}_{50}$ values were $8.37 \pm 0.14$ and $91.34 \pm 1.98 \mu \mathrm{g} / \mathrm{mL}$ for $\mathrm{DPPH}^{\circ}$ and superoxide anion radicals, respectively.

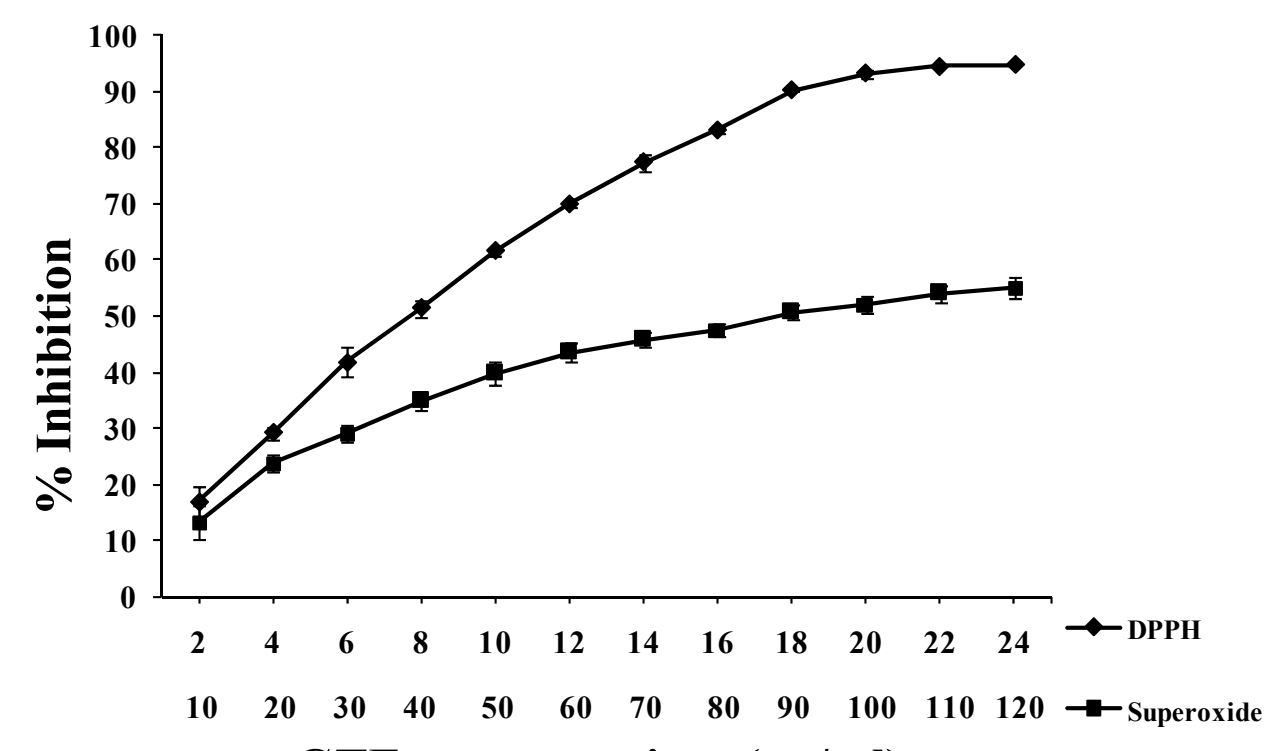

GTE concentrations $(\mu \mathrm{g} / \mathrm{ml})$

\subsection{GTE Reduces the Stress-Induced hsp-16-2 Expression in C. elegans}

Small heat shock proteins (sHSPs) are biomarkers for the exposure to thermal and oxidative stressors, and they are highly inducible during exposure to pro-oxidants, such as juglone [53]. sHSPs act as molecular chaperones by preventing the accumulation of aggregated proteins [64]. The TJ375 strain has a GFP reporter controlled by the $h s p-16.2$ promoter [53] that is not active under standard conditions. However, after exposure to oxidative stress, $h s p-16.2$ becomes induced, and the worms exhibit an extensive GFP expression in the pharynx (Figure 4). In the present study, the TJ375 strain is used to figure out the protective effect of GTE against oxidative stress produced by treatment with 
nonlethal dose of juglone. The expression of $h s p-16.2 / G F P$ (induced by $20 \mu \mathrm{M}$ juglone) was suppressed by $68.43 \%$ in worms fed with $100 \mu \mathrm{g} / \mathrm{mL}$ of GTE, as compared to controls (control: GFP mean pixel density of $1,621.644 \pm 133.22$ vs. GTE, GFP mean pixel density of $512.01 \pm 126.89$, $p<0.001$; Figure 4). These results indicate that GTE is bioavailable and able to increase the stress resistance in C. elegans.

Figure 4. C. elegans strain TJ375 (hsp-16.2/GFP) visualizes the induction of the hsp-16.2/GFP reporter in response to juglone treatment. Experimental worms were cultured in liquid medium and treated without (a) and with (b) $100 \mu \mathrm{g} / \mathrm{mL}$ GTE for $48 \mathrm{~h}$ followed by $20 \mu \mathrm{M}$ juglone (juglone treatment was in new medium) for $24 \mathrm{~h}$. (c) The quantitation of the GFP in response to oxidative stress. The GFP fluorescence is not detectable with GTE alone or with no juglone treatment. Comparisons between treatments and controls were significant using the two-tailed, unpaired Student's $t$-test. Data were obtained from three independent experiments with 25 worms in each group. *** $p<0.001$.

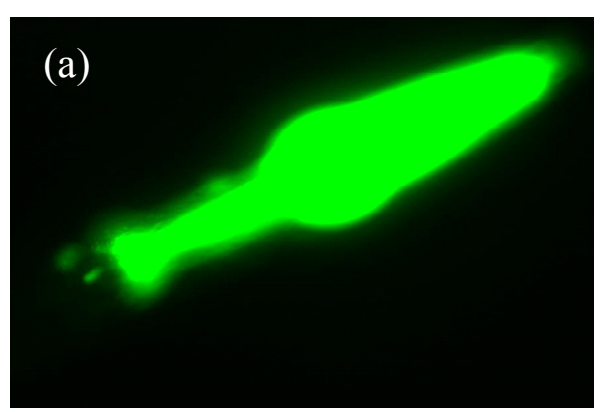

Control

$20 \mu \mathrm{M}$ Juglone

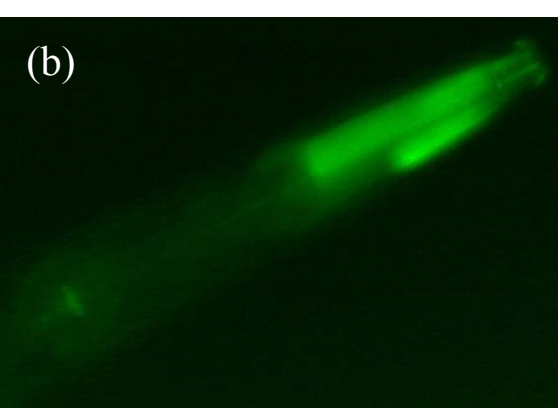

$100 \mu \mathrm{g} / \mathrm{ml} \mathrm{GTE}$

$+20 \mu \mathrm{M}$ Juglone

(c)

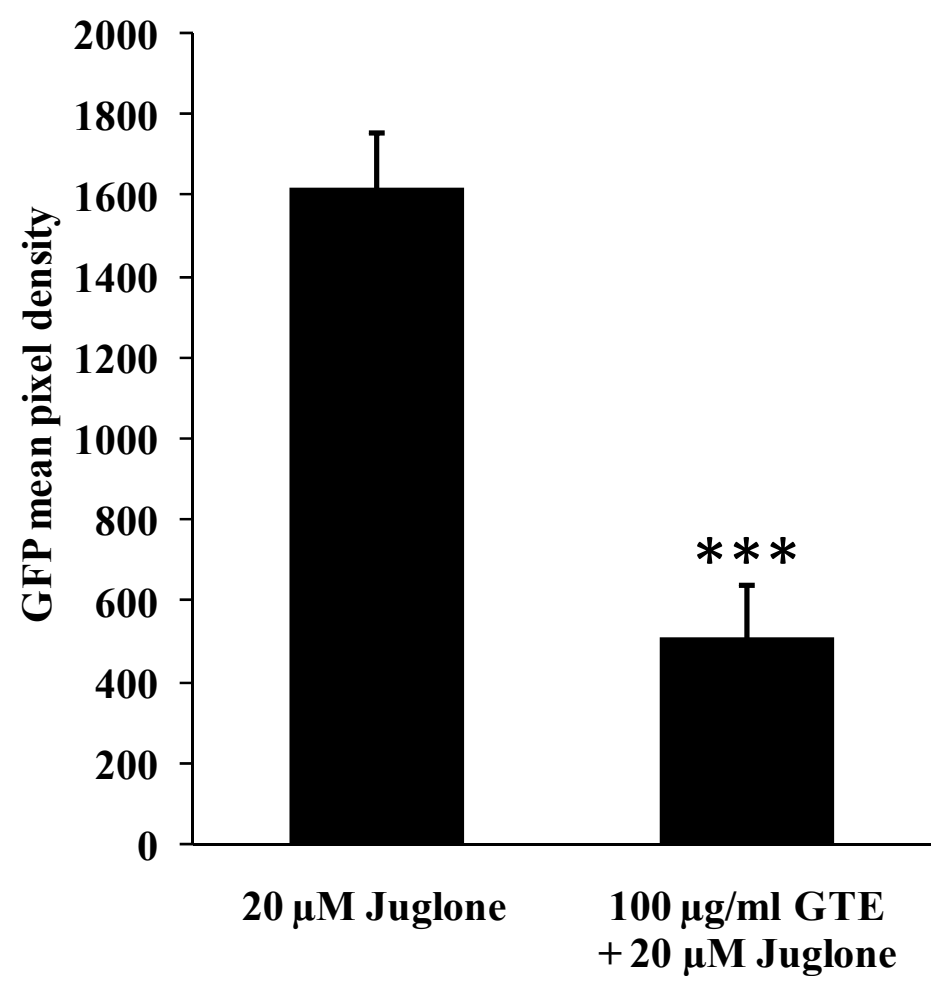




\subsection{GTE Increases Survival Rate in C. elegans}

In this experiment, $\mathrm{N} 2$ worms were pretreated with $100 \mu \mathrm{g} / \mathrm{mL}$ GTE for $48 \mathrm{~h}$ and then transferred to new medium and exposed to a lethal dose of juglone $(80 \mu \mathrm{M})$ for $24 \mathrm{~h}$, after which the alive worms were counted. The results (Figure 5) showed that the pretreatment of the N2 worms with $100 \mu \mathrm{g} / \mathrm{mL}$ GTE is indeed able to protect the organism against lethal oxidative stress. The average of surviving worms after treatment with $80 \mu \mathrm{M}$ juglone was $32.43 \pm 1.71 \%$ and for the group pretreated with GTE for $48 \mathrm{~h}$ was $80.74 \pm 3.51 \%$ (the increase rate was $48.31 \%, p<0.001$, Figure 5).

Figure 5. Survival rates of wild-type $C$. elegans under an acute lethal dose of juglone. In this experiment, the worms were cultured in liquid medium and treated with $100 \mu \mathrm{g} / \mathrm{mL}$ GTE for $48 \mathrm{~h}$ followed with $80 \mu \mathrm{M}$ juglone for $24 \mathrm{~h}$ (juglone treatment was in new medium). The mean percentage of live worms was calculated from three independent experiments. Comparisons between treatments and controls were significant using the two-tailed, unpaired, Student's $t$-test. Data were obtained from three independent trials, each consisting of at least 150 worms. ${ }^{* * *} p<0.001$.

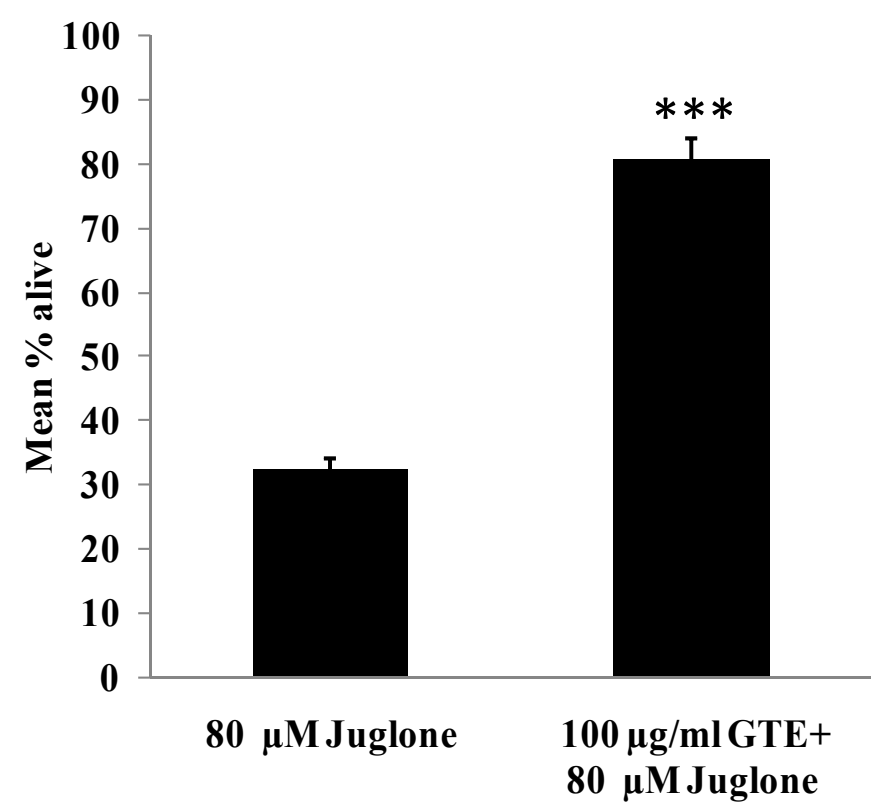

The treatment of worms with EGCG showed similar results by upregulating the aging-associated genes, such as daf-16, sod-3, and skn-1 [65]. Compared with EGCG, the mode of action of GTE seems to be similar, since EGCG activates the daf2/insulin-signaling pathway by the induction of DAF-16 translocation from cytoplasm into nucleus in the TJ356 (DAF-16/GFP) strain [37]. The inhibition of daf-2, a homologue of the insulin receptor, leads also to nuclear localization of DAF-16/GFP [66]. DAF-16/FOXO is a transcription factor that promotes longevity and stress resistance, including antioxidative enzymes, like superoxide dismutase and catalase [67]. In addition, several studies showed that polyphenols can enhance oxidative stress resistance and prolong the lifespan in C. elegans, probably by modulation of the daf2/insulin-signaling pathway [37,40]. 


\section{Conclusions}

Catechins represent $30 \%$ of the dry weight of green tea leaves and are the active components of GTE, which exhibits considerable antioxidant activities in vitro and in vivo. In C. elegans, GTE reduces the oxidative stress and elevates the stress resistance by attenuating the heat shock protein (hsp-16.2) response and by increasing the survival rate after exposition to a lethal dose of the pro-oxidant, juglone. Our results imply that the epidemiological data, which see the beneficial effects of green tea consumption with good health and the reduction of the risk of several diseases related to oxidative stress, have a rational base.

\section{Acknowledgments}

We gratefully acknowledge the support of U. Engel and C. Ackermann (Nikon Imaging Centre, Heidelberg University). The Caenorhabditis Genetics Center (Minnesota University) kindly supplied the worm strains. S. Galas (Montpellier) and Christopher D. Link (Colorado University) provided valuable advice and suggestions in the early phase of our experiments.

\section{Conflicts of Interest}

The authors declare no conflict of interest.

\section{References}

1. Dalle-Donne, I.; Rossi, R.; Colombo, R.; Giustarini, D.; Milzani, A. Biomarkers of oxidative damage in human disease. Clin. Chem. 2006, 52, 601-623.

2. Jenner, P. Oxidative stress in parkinson's disease. Ann. Neurol. 2003, 53, 26-28.

3. Valko, M.; Rhodes, C.J.; Moncol, J.; Izakovic, M.; Mazur, M. Free radicals, metals and antioxidants in oxidative stress-induced cancer. Chem. Biol. Interact. 2006, 160, 1-40.

4. Valko, M.; Leibfritz, D.; Moncol, J.; Cronin, M.T.; Mazur, M.; Telser, J. Free radicals and antioxidants in normal physiological functions and human disease. Int. J. Biochem. Cell. Biol. 2007, 39, 44-84.

5. Valko, M.; Izakovic, M.; Mazur, M.; Rhodes, C.J.; Telser, J. Role of oxygen radicals in DNA damage and cancer incidence. Mol. Cell. Biochem. 2004, 266, 37-56.

6. Van Wyk, B.A.; Wink, M. Medicinal Plants of the World; Timber Press: Portland, OR, USA, 2004.

7. Miketova, P.; Schram, K.H.; Whitney, J.; Li, M.; Huang, R.; Kerns, E.; Valcic, S.; Timmermann, B.N.; Rourick, R.; Klohr, S. Tandem mass spectrometry studies of green tea catechins. Identification of three minor components in the polyphenolic extract of green tea. J. Mass Spectrom. 2000, 35, 860-869.

8. Graham, H.N. Green tea composition, consumption, and polyphenol chemistry. Prev. Med. 1992, 21, 334-350.

9. Jagtap, S.; Meganathan, K.; Wagh, V.; Winkler, J.; Hescheler, J.; Sachinidis, A. Chemoprotective mechanism of the natural compounds, epigallocatechin-3-o-gallate, quercetin and curcumin against cancer and cardiovascular diseases. Curr. Med. Chem. 2009, 16, 1451-1462. 
10. Wolfram, S. Effects of green tea and EGCG on cardiovascular and metabolic health. J. Am. Coll. Nutr. 2007, 26, 373-388.

11. Nagle, D.G.; Ferreira, D.; Zhou, Y. Epigallocatechin-3-gallate (EGCG): Chemical and biomedical perspectives. Phytochemistry 2006, 67, 1849-1855.

12. Johnson, J.J.; Bailey, H.H.; Mukhtar, H. Green tea polyphenols for prostate cancer chemoprevention: A translational perspective. Phytomedicine 2010, 17, 3-13.

13. Chow, H.H.; Cai, Y.; Hakim, I.A.; Crowell, J.A.; Shahi, F.; Brooks, C.A.; Dorr, R.T.; Hara, Y.; Alberts, D.S. Pharmacokinetics and safety of green tea polyphenols after multiple-dose administration of epigallocatechin gallate and polyphenon E in healthy individuals. Clin. Cancer Res. 2003, 9, 3312-3319.

14. Chow, H.H.; Hakim, I.A.; Vining, D.R.; Crowell, J.A.; Ranger-Moore, J.; Chew, W.M.; Celaya, C.A.; Rodney, S.R.; Hara, Y.; Alberts, D.S. Effects of dosing condition on the oral bioavailability of green tea catechins after single-dose administration of polyphenon $\mathrm{E}$ in healthy individuals. Clin. Cancer Res. 2005, 11, 4627-4633.

15. Hong, J.; Lu, H.; Meng, X.; Ryu, J.H.; Hara, Y.; Yang, C.S. Stability, cellular uptake, biotransformation, and efflux of tea polyphenol (-)-epigallocatechin-3-gallate in ht-29 human colon adenocarcinoma cells. Cancer Res. 2002, 62, 7241-7246.

16. Zaveri, N.T. Green tea and its polyphenolic catechins: Medicinal uses in cancer and noncancer applications. Life Sci. 2006, 78, 2073-2080.

17. Cooper, R.; Morre, D.J.; Morre, D.M. Medicinal benefits of green tea: Part i. Review of noncancer health benefits. J. Altern. Complement. Med. 2005, 11, 521-528.

18. Bettuzzi, S.; Brausi, M.; Rizzi, F.; Castagnetti, G.; Peracchia, G.; Corti, A. Chemoprevention of human prostate cancer by oral administration of green tea catechins in volunteers with high-grade prostate intraepithelial neoplasia: A preliminary report from a one-year proof-of-principle study. Cancer Res. 2006, 66, 1234-1240.

19. Wang, Z.Y.; Cheng, S.J.; Zhou, Z.C.; Athar, M.; Khan, W.A.; Bickers, D.R.; Mukhtar, H. Antimutagenic activity of green tea polyphenols. Mutat. Res. 1989, 223, 273-285.

20. Santhosh, K.T.; Swarnam, J.; Ramadasan, K. Potent suppressive effect of green tea polyphenols on tobacco-induced mutagenicity. Phytomedicine 2005, 12, 216-220.

21. Mehrabian, S. The study of antioxidant and anticarcinogenic green tea and black tea. Pak. J. Biol. Sci. 2007, 10, 989-991.

22. Kuroda, Y.; Hara, Y. Antimutagenic and anticarcinogenic activity of tea polyphenols. Mutat. Res. 1999, 436, 69-97.

23. De Mejia, E.G.; Ramirez-Mares, M.V.; Puangpraphant, S. Bioactive components of tea: Cancer, inflammation and behavior. Brain Behav. Immun. 2009, 23, 721-731.

24. Stendell-Hollis, N.R.; Thomson, C.A.; Thompson, P.A.; Bea, J.W.; Cussler, E.C.; Hakim, I.A. Green tea improves metabolic biomarkers, not weight or body composition: A pilot study in overweight breast cancer survivors. J. Hum. Nutr. Diet. 2010, 23, 590-600.

25. Basu, A.; Sanchez, K.; Leyva, M.J.; Wu, M.; Betts, N.M.; Aston, C.E.; Lyons, T.J. Green tea supplementation affects body weight, lipids, and lipid peroxidation in obese subjects with metabolic syndrome. J. Am. Coll. Nutr. 2010, 29, 31-40.

26. Yang, C.S.; Wang, Z.Y. Tea and cancer. J. Natl. Cancer Inst. 1993, 85, 1038-1049. 
27. Abbas, S.; Wink, M. Epigallocatechin gallate from green tea (Camellia sinensis) increases lifespan and stress resistance in Caenorhabditis elegans. Planta Med. 2009, 75, 216-221.

28. Wink, M.; Abbas, S. Epigallocatechin Gallate (EGCG) from Green Tea (Camellia sinensis) and Other Natural Products Mediate Stress Resistance and Slows down Aging Processes in Caenorhabditis elegans. In Tea in Health and Disease Prevention; Preedy, V.R., Ed.; Elsevier: London, UK, 2013; pp. 1105-1116.

29. Wink, M. Evolutionary advantage and molecular modes of action of multi-component mixtures used in phytomedicine. Curr. Drug Metab. 2008, 9, 996-1009.

30. Wink, M.; Van Wyk, B.E. Mind-Altering and Poisonous Plants of the World; Timber Press: London, UK, 2008.

31. Wink, M.; Schimmer, O. Molecular Modes of Action of Defensive Secondary Metabolites. In Annual Plant Reviews: Functions and Biotechnology of Plant Secondary Metabolites; Wink, M., Ed.; Wiley-Blackwell: Oxford, UK, 2009; Volume 39, pp. 21-161.

32. Brenner, S. The genetics of Caenorhabditis elegans. Genetics 1974, 77, 71-94.

33. Sonnhammer, E.L.; Durbin, R. Analysis of protein domain families in Caenorhabditis elegans. Genomics 1997, 46, 200-216.

34. Kaletta, T.; Hengartner, M.O. Finding function in novel targets: C. elegans as a model organism. Nat. Rev. Drug Discov. 2006, 5, 387-398.

35. Brenner, S. Foreward. In The Nematode Caenorhabditis elegans; Wood, W.B., Ed.; Cold Spring Harbor Laboratory Press: Cold Spring Harbor, NY, USA, 1988; pp. ix-xiii.

36. Nanjo, F.; Mori, M.; Goto, K.; Hara, Y. Radical scavenging activity of tea catechins and their related compounds. Biosci. Biotechnol. Biochem. 1999, 63, 1621-1623.

37. Abbas, S.; Wink, M. Epigallocatechin gallate inhibits beta amyloid oligomerization in Caenorhabditis elegans and affects the daf-2/insulin-like signaling pathway. Phytomedicine 2010, 17, 902-909.

38. Saul, N.; Pietsch, K.; Menzel, R.; Sturzenbaum, S.R.; Steinberg, C.E. Catechin induced longevity in C. elegans: From key regulator genes to disposable soma. Mech. Ageing Dev. 2009, 130, 477-486.

39. Kirkwood, T.B. Evolution of ageing. Nature 1977, 270, 301-304.

40. Chen, W.; Muller, D.; Richling, E.; Wink, M. Anthocyanin-rich purple wheat prolongs the life span of Caenorhabditis elegans probably by activating the daf-16/foxo transcription factor. J. Agric. Food Chem. 2013, 61, 3047-3053.

41. Chen, W.; Rezaizadehnajafi, L.; Wink, M. Influence of resveratrol on oxidative stress resistance and life span in Caenorhabditis elegans. J. Pharm. Pharmacol. 2013, 65, 682-688.

42. Chen, W.; Sudji, I.R.; Wang, E.; Joubert, E.; van Wyk, B.E.; Wink, M. Ameliorative effect of aspalathin from rooibos (Aspalathus linearis) on acute oxidative stress in Caenorhabditis elegans. Phytomedicine 2013, 20, 380-386.

43. Wilson, M.A.; Shukitt-Hale, B.; Kalt, W.; Ingram, D.K.; Joseph, J.A.; Wolkow, C.A. Blueberry polyphenols increase lifespan and thermotolerance in Caenorhabditis elegans. Aging Cell 2006, 5, 59-68. 
44. Solomon, A.; Bandhakavi, S.; Jabbar, S.; Shah, R.; Beitel, G.J.; Morimoto, R.I. Caenorhabditis elegans osr-1 regulates behavioral and physiological responses to hyperosmotic environments. Genetics 2004, 167, 161-170.

45. Wu, Z.; Smith, J.V.; Paramasivam, V.; Butko, P.; Khan, I.; Cypser, J.R.; Luo, Y. Ginkgo biloba extract egb 761 increases stress resistance and extends life span of Caenorhabditis elegans. Cell. Mol. Biol. (Noisy-le-grand) 2002, 48, 725-731.

46. Evason, K.; Collins, J.J.; Huang, C.; Hughes, S.; Kornfeld, K. Valproic acid extends Caenorhabditis elegans lifespan. Aging Cell 2008, 7, 305-317.

47. Sulston, J.; Hodgkin, J. The Nematode Caenorhabditis elegans; Cold Spring Harbor Laboratory Press: New York, NY, USA, 1988; pp. 587-606.

48. Blois, M.S. Antioxidant determinations by the use of a stable free radical. Nature 1958, 26, 1199-1200.

49. Brand-Williams, W.; Cuvelier, M.E.; Berset, C. Use of a free radical method to evaluate antioxidant activity. LWT-Food Sci. Technol. 1995, 28, 25-30.

50. Bondet, V.; Brand-Williams, W.; Berset, C. Kinetics and mechanisms of antioxidant activity using the DPPH ${ }^{*}$ free radical method. LWT-Food Sci. Technol. 1997, 30, 609-615.

51. Molyneux, P. The use of the stable free radical diphenylpicrylhydrazyl (DPPH) for estimating antioxidant activity. J. Sci. Technol. 2004, 26, 211-219.

52. Robak, J.; Gryglewski, R.J. Flavonoids are scavengers of superoxide anions. Biochem. Pharmacol. 1988, 37, 837-841.

53. Link, C.D.; Cypser, J.R.; Johnson, C.J.; Johnson, T.E. Direct observation of stress response in Caenorhabditis elegans using a reporter transgene. Cell Stress Chaperones 1999, 4, 235-242.

54. Miketova, P.; Schram, K.H.; Whitney, J.L.; Kerns, E.H.; Valcic, S.; Timmermann, B.N.; Volk, K.J. Mass spectrometry of selected components of biological interest in green tea extracts. J. Nat. Prod. 1998, 61, 461-467.

55. Poon, G.K. Analysis of catechins in tea extracts by liquid chromatography-electrospray ionization mass spectrometry. J. Chromatogr. A 1998, 794, 63-74.

56. Bastos, D.H.; Saldanha, L.A.; Catharino, R.R.; Sawaya, A.C.; Cunha, I.B.; Carvalho, P.O.; Eberlin, M.N. Phenolic antioxidants identified by esi-ms from yerba mate (Ilex paraguariensis) and green tea (Camellia sinensis) extracts. Molecules 2007, 12, 423-432.

57. Dhalla, N.S.; Temsah, R.M.; Netticadan, T. Role of oxidative stress in cardiovascular diseases. J. Hypertens. 2000, 18, 655-673.

58. Sayre, L.M.; Smith, M.A.; Perry, G. Chemistry and biochemistry of oxidative stress in neurodegenerative disease. Curr. Med. Chem. 2001, 8, 721-738.

59. Harman, D. Aging: A theory based on free radical and radiation chemistry. J. Gerontol. A Biol. Sci. Med. Sci. 1956, 11, 298-300.

60. Hu, C.; Kitts, D.D. Evaluation of antioxidant activity of epigallocatechin gallate in biphasic model systems in vitro. Mol. Cell. Biochem. 2001, 218, 147-155.

61. Nanjo, F.; Goto, K.; Seto, R.; Suzuki, M.; Sakai, M.; Hara, Y. Scavenging effects of tea catechins and their derivatives on 1,1-diphenyl-2-picrylhydrazyl radical. Free Radic. Biol. Med. 1996, 21, 895-902. 
62. Leung, L.K.; Su, Y.; Chen, R.; Zhang, Z.; Huang, Y.; Chen, Z.Y. Theaflavins in black tea and catechins in green tea are equally effective antioxidants. J. Nutr. 2001, 131, 2248-2251.

63. Yokozawa, T.; Nakagawa, T.; Kitani, K. Antioxidative activity of green tea polyphenol in cholesterol-fed rats. J. Agric. Food Chem. 2002, 50, 3549-3552.

64. Leroux, M.R.; Melki, R.; Gordon, B.; Batelier, G.; Candido, E.P. Structure-function studies on small heat shock protein oligomeric assembly and interaction with unfolded polypeptides. J. Biol. Chem. 1997, 272, 24646-24656.

65. Zhang, L.; Jie, G.; Zhang, J.; Zhao, B. Significant longevity-extending effects of EGCG on Caenorhabditis elegans under stress. Free Radic. Biol. Med. 2009, 46, 414-421.

66. Henderson, S.T.; Johnson, T.E. Daf-16 integrates developmental and environmental inputs to mediate aging in the nematode Caenorhabditis elegans. Curr. Biol. 2001, 11, 1975-1980.

67. Oh, S.W.; Mukhopadhyay, A.; Dixit, B.L.; Raha, T.; Green, M.R.; Tissenbaum, H.A. Identification of direct daf-16 targets controlling longevity, metabolism and diapause by chromatin immunoprecipitation. Nat. Genet. 2006, 38, 251-257.

(C) 2014 by the authors; licensee MDPI, Basel, Switzerland. This article is an open access article distributed under the terms and conditions of the Creative Commons Attribution license (http://creativecommons.org/licenses/by/3.0/). 